\title{
Multiple Strokes in a Latin American Patient
}

\author{
Ramez Halaseh $^{a}$ Malik Shehadeh $^{a}$ Rachel Marcus $^{b}$ \\ aInternal Medicine Department, Medstar Washington Hospital Center, Washington, DC, \\ USA; ${ }^{b}$ Cardiology Department, Medstar Washington Hospital Center, Medstar Heart and \\ Vascular Institute, Washington, DC, USA
}

\author{
Keywords \\ Stroke $\cdot$ Chagas cardiomyopathy $\cdot$ Trypanosoma cruzi
}

\begin{abstract}
We present a case of a recent immigrant from El Salvador without past medical history who presented to our hospital with symptoms concerning for acute stroke. Brain magnetic resonance imaging (MRI) with gadolinium confirmed an acute stroke along with multiple prior infarcts involving different vascular beds. Head magnetic resonance arteriogram did not reveal any occlusions/stenosis or aneurysmal changes. His subsequent extensive evaluation included an electrocardiogram (ECG) that revealed bifascicular block and echocardiography that suggested an apical aneurysm, but images were limited to assess. To further assess the likelihood of cardiac embolism, he underwent cardiac MRI with gadolinium, which confirmed the apical aneurysm. Because of his country of origin and classic ECG and echo findings, Chagas disease was suspected, and both commercial ELISA and confirmatory ELISA and TESA blots were positive. This is both a classic presentation of Chagas cardiomyopathy and an important reminder that Chagas disease should be considered in the differential diagnosis of cardioembolic stroke in Latin American immigrants from an endemic country.
\end{abstract}

\section{Introduction}

Chagas disease is a chronic parasitic infection caused by the protozoan Trypanosoma cruzi that is transmitted to humans by triatomine insects in endemic areas of Latin America $[1,2]$. The disease affects about 6 million people in North and South America and is currently considered the most common cause of nonischemic cardiomyopathy in endemic Latin American countries [1]. Ischemic stroke may be the first manifestation of chronic Chagas 
Fig. 1. Initial brain MRI with gadolinium showing an acute infarct measuring $4 \times 1 \mathrm{~cm}$ in the right middle frontal gyrus of the MCA territory. MRI, magnetic resonance imaging; MCA, middle cerebral artery.

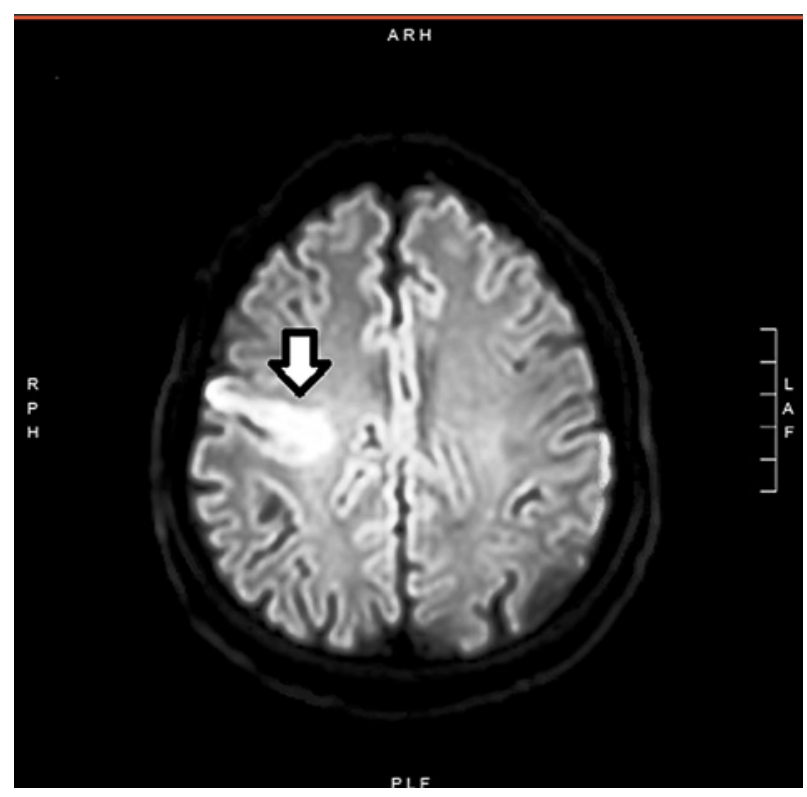

cardiomyopathy (CCC) [3] and should be suspected in any patient from an endemic Latin American country presenting with cardioembolic stroke.

\section{Case Report/Case Presentation}

A 56-year-old El-Salvadorian male with no past medical history presented to our hospital with new-onset left-sided weakness involving the upper and lower extremities with difficulty speaking of $2 \mathrm{~h}$ duration. Upon arrival at the hospital, his vital signs were unremarkable. On physical examination, he scored 9 points on the NIH stroke scale (left facial droop, left upper extremity weakness, left lower extremity weakness, left-sided paresthesia, dysarthria, and extinction to bilateral simultaneous stimulation). Laboratory investigations showed LDL of $115 \mathrm{mg} / \mathrm{dL}$ and a normal hemoglobin A1C. Brain magnetic resonance imaging (MRI) with gadolinium showed an acute infarct in the right middle cerebral artery territory (shown in Fig. 1) along with old cortical infarcts in the left frontal, parietal, and occipital lobes and an old left cerebellar infarct (shown in Fig. 2). Brain magnetic resonance arteriogram without contrast showed no large vessel occlusion/stenosis or aneurysmal changes. The patient subsequently received intravenous alteplase and was admitted to the hospital for monitoring and further stroke workup. His electrocardiogram (ECG) showed normal sinus rhythm with bifascicular block. Carotid duplex was unremarkable. A 2D echocardiogram was obtained and demonstrated borderline reduced left ventricular ejection fraction of 50-55\%; the apex was not well imaged, and no signs for left ventricular hypertrophy were seen. Repeat echocardiogram with contrast suggested a possible focal apical aneurysm, but the study was suboptimal to assess. Because of the suboptimal apical imaging by echocardiogram, cardiac MRI with gadolinium was obtained and showed near transmural late gadolinium enhancement of the lateral wall with a focal apical aneurysm (shown in Fig. 3). Laboratory assessment for thrombophilia was negative, and an implantable loop recorder was placed to assess for occult atrial fibrillation. Upon review of his clinical picture, country of origin, and imaging studies, his risk for CCC became apparent, and Trypanosoma cruzi IgG testing was performed. Both commercial serologic testing and CDC confirmation with ELISA and TESA blot assays 
Fig. 2. Initial brain MRI with gadolinium showing an old left cerebellar infarct. MRI, magnetic resonance imaging.

Fig. 3. Cardiac MRI with gadolinium showing evidence of an apical aneurysm with regional thinning and transmural gadolinium enhancement. MRI, magnetic resonance imaging.
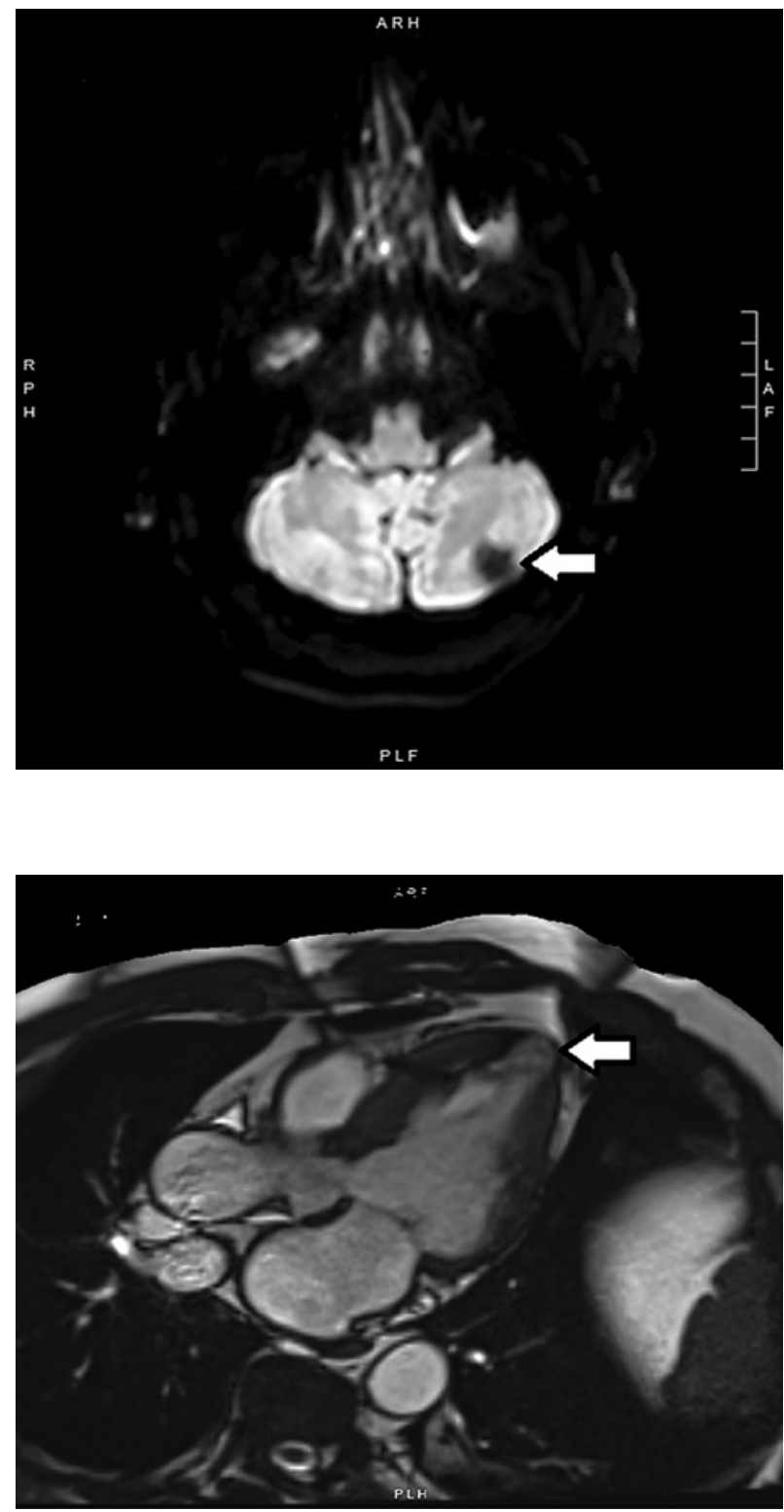

confirmed this diagnosis. By the end of his hospitalization, his neurological symptoms resolved except for a mild left facial droop.

\section{Discussion/Conclusion}

Chagas cardiomyopathy typically manifests $15-30$ years after initial infection, and $20-30 \%$ of infected individuals can develop various cardiac manifestations such as ventricular arrhythmias, cardioembolic strokes, heart failure, or sudden cardiac death [2]. Strokes in CCC patients can originate from a left ventricular aneurysm with associated thrombus, even when the EF is preserved, and atrial fibrillation is also common in CCC patients [3]. In a heart failure clinic in Brazil, patients with CCC were at higher risk for stroke than patients with other cardiomyopathies (15 vs. 6.3\%), and CCC patients have fewer risk factors and higher rates of recurrent 
stroke than other patients with stroke [3]. Strokes in CCC typically involve the middle cerebral artery in up to $80 \%$ of cases [4]. The diagnosis of Chagas disease is made by serologic testing, which must include 2 different serologic assays. ECG abnormalities can indicate the presence of CCC, and they include right bundle branch block, left anterior fascicular block, bifascicular block, atrial fibrillation, first-degree AV block, and premature ventricular complexes [2]. Latin American immigrants to the US from Chagas endemic countries have impressive rates of CCC: $5 \%$ of patients with conduction abnormalities and $18 \%$ of patients with bifascicular block had CCC [5]. In one study of patients with nonischemic cardiomyopathy in US clinics, $19 \%$ of individuals from endemic countries were found to have CCC as the cause of their heart failure [6]. Beyond an ECG, further evaluation is usually required in patients with suspected CCC. In such patients, echocardiogram can show regional wall motion abnormalities, especially in the basal inferolateral wall, with an apical aneurysm/thrombus that can be hard to visualize without careful imaging or the use of an echo contrast agent [7]. This is critically important in that up to $47 \%$ of CCC patients with ischemic stroke will have this lesion, and its timely identification can obviate the need for evaluation for other source of thromboembolism [8]. Cardiac MRI should be considered in a stroke patient with suspected Chagas disease if the presence of an apical aneurysm cannot be determined by echocardiogram [9].

\section{Acknowledgements}

We would like to dedicate this case report to our family and friends who supported us throughout our journey in the field of medicine.

\section{Statement of Ethics}

The patient described in this article has given written, informed consent to publish his case, radiographic images, and pathological reports. The research in this article was conducted ethically in accordance with the World Medical Association Declaration of Helsinki. The study is exempt from Ethical Committee approval as per the guideline of MedStar Health Research Institute given that it is a case report and informed consent was obtained.

\section{Conflict of Interest Statement}

The authors have no conflicts of interest to declare.

\section{Funding Sources}

No funding sources to be reported.

\section{Author Contributions}

Ramez Halaseh, Malik Shehadeh, and Rachel Marcus have contributed to literature review and drafting the manuscript for intellectual content. Dr. Marcus was the supervising attending and helped guide the structuring of the case report and making sure that the contents are up to date with the most recent data.

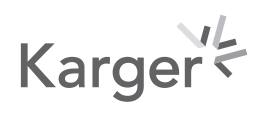




\section{References}

1 Rassi A Jr, Rassi A, Marin-Neto JA. Chagas heart disease: pathophysiologic mechanisms, prognostic factors and risk stratification. Mem Inst Oswaldo Cruz. 2009 Jul;104(Suppl 1):152-8.

2 Rassi A Jr, Rassi A, Marin-Neto JA. Chagas disease. Lancet. 2010 Apr 17;375(9723):1388-402.

3 Oliveira-Filho J, Viana LC, Vieira-de-Melo RM, Faiçal F, Torreão JA, Villar FA, et al. Chagas disease is an independent risk factor for stroke: baseline characteristics of a Chagas disease cohort. Stroke. 2005 Sep;36(9): 2015-7.

4 Carod-Artal FJ, Vargas AP, Horan TA, Nunes LG. Chagasic cardiomyopathy is independently associated with ischemic stroke in Chagas disease. Stroke. 2005 May 1;36(5):965-70.

5 Traina MI, Hernandez S, Sanchez DR, Dufani J, Salih M, Abuhamidah AM, et al. Prevalence of Chagas disease in a U.S. population of Latin American immigrants with conduction abnormalities on electrocardiogram. PLoS Negl Trop Dis. 2017 Jan 5;11(1):e0005244.

6 Traina MI, Sanchez DR, Hernandez S, Bradfield JS, Labedi MR, Ngab TA, et al. Prevalence and impact of chagas disease among Latin American immigrants with nonischemic cardiomyopathy in Los Angeles, California. Circ Heart Fail. 2015 Sep;8(5):938-43.

7 Acquatella H. Echocardiography in Chagas heart disease. Circulation. 2007 Mar 6;115(9):1124-31.

8 Nunes MdoC, Barbosa MM, Rocha MO. Peculiar aspects of cardiogenic embolism in patients with Chagas' cardiomyopathy: a transthoracic and transesophageal echocardiographic study. J Am Soc Echocardiogr. 2005 Jul;18(7):761-7.

9 Schmidt A, Maciel BC, Marin-Neto JA. Clinical trial report: time is resonant for right ventricular evaluation. Curr Cardiovasc Imaging Rep. 2011;4(3):177-9. 